\title{
The Biases in Flavonoids and Anthocyanin Biosynthesis of a Red Flesh Table Grape Revealed by Metabolome and Transcriptome Co-analysis
}

renxiang lu

China Agricultural University https://orcid.org/0000-0002-5390-7189

Miaoyu Song

China Agricultural University

Zhe Wang

China Agricultural University

Yanlei Zhai

China Agricultural University

Chaoyang Hu

China Agricultural University

Avihai Perl

Agricultural Research Organization Volcani Center

Huiqin Ma ( $\nabla$ hqma@cau.edu.cn )

China Agricultural University https://orcid.org/0000-0002-2488-3958

\section{Research Article}

Keywords: red-flesh grape, transcriptome, metabolomics, anthocyanin, flavonoids

Posted Date: December 20th, 2021

DOI: https://doi.org/10.21203/rs.3.rs-1139881/v1

License: (9) This work is licensed under a Creative Commons Attribution 4.0 International License.

Read Full License 


\section{Abstract}

Red flesh is a welcomed fruit trait, yet the regulation of red flesh formation in grape is not well understood. 'Mio Red' is a seedless table grape variety with light red flesh and blue-purple skin, the flesh color developed in the late stage of berry ripening, remarkably later than the skin coloring at veraison. The flesh and skin flavonoids metabolome and the transcriptome were analyzed. A total of 173 flavonoids including 17 anthocyanins were identified, 68 were found significantly different (Fold change $\geq 2$ or $\leq 0$. 5, VIP $\geq 1$ ). Quercetin 3-0-glucoside, epicatechin-epiafzelechin, apigenin 6,8-C-diglucoside and hesperetin 5-O-glucoside were of higher content in the flesh, while the rest flavonoids were of higher content in the skin. The main anthocyanin in the flesh was pelargonidin derivatives in contrast to peonidin derivatives in the skin. Transcriptome comparison recruited 3970 differentially expressed genes (DEGs, log2Fold change $>=1, F D R<0.05, F P K M \geq 1$ ), among them 57 were structural genes of flavonoid metabolism pathway. Two anthocyanin synthase (ANS) DEGs were annotated, ANS1 (Vitvi11g00565) and ANS2 (Vitvi02g00435) led the expression in the flesh and skin respectively. In the flesh, anthocyanin biosynthesis structural gene UFGT, positive regulators $M Y B A 1 / 2 / 3$, and anthocyanin transporters GST14 and MATE5 were of significantly lower expression, while negative regulators $M Y B C 2-L 1$ and $M Y B 3$ were of higher transcription. The results of this study provide new information in the coloring mechanism of red flesh grape and assisting breeding of future table grapes having higher content of phytonutrient providing the health benefit as red wines.

\section{Key Message}

Metabolome and transcriptome co-analysis revealed independently and differentially regulated flavonoids and anthocyanin biosynthesis pathway and key genes in the flesh and skin of a new red flesh table grape variety.

\section{Introduction}

Grape is one of the most important fruits in the world, has been recorded several thousands of varieties. In 2019, the planting area was 69.3 hectares (ha), and the production reached 77.14 million tons (http://www.fao.org/faostat/zh/ \# data/QC), China is the largest table grape producer and consumer. Grape berry development follows a typical double sigmoid growth curve. Veraison, the start of stage III, is marked by berry softening and skin color change (Coombe and Bishop, 1980). According to the skin color, grape varieties can be divided into two major categories, red grapes which are of purple, red or pink appearance, and white grapes which are of light green to golden color at ripening.

Flavonoids are a kind of secondary metabolites including flavonoids, flavanones, proanthocyanidins and anthocyanidins, they are reactive oxygen species scavengers in plants, and have high value in human health (Agati et al., 2012). Anthocyanin is the most important flavonoids in grape berries determining berry color. Six anthocyanins are commonly identified in grape berries, namely cyanidin, peonidin, delphinin, malvidin, pelargonidin and petunidin (Fuleki and Ricardo-da-Silva, 1997). The dominant 
anthocyanin is different among grape varieties, and pelargonidin derivatives can hardly be detected in most of Vitis vinifera varieties (Ageorges et al., 2006).

Anthocyanin biosynthesis via phenylpropane pathway and flavonoid biosynthesis pathway and the main regulating transcription factors have been widely elucidated with grape skin (Jiu et al., 2021).

Phenylalanine ammonia lyase (PAL), cinnamate 4-hydroxylase $(\mathrm{C} 4 \mathrm{H})$ and 4-coumaroyl-CoA synthase $(4 \mathrm{CL})$ are the key members of the phenylpropane synthesis pathway; chalcone synthase (CHS), chalcone isomerase $(\mathrm{CHI})$, flavanone 3-hydroxylase $(\mathrm{F} 3 \mathrm{H})$, flavono-3'-hydroxylase $\left(\mathrm{F} 3{ }^{\prime} \mathrm{H}\right)$, flavonoid-3', 5'-hydroxylase (F3'5'H), dihydroflavonol-4-reductase (DFR), anthocyanin synthase (ANS) and UDP-Glc:flavonoid-3-Oglucosyltransferase (UFGT) compose the route to anthocyanin, while other enzymes, such as flavone synthase (FNS) and flavonol synthase (FLS) catalyzing the direct production of flavonoids and flavonols from flavonoid intermediates; leucoanthocyanidin reductase (LAR) and anthocyanin reductase (ANR) catalyze the synthesis of catechins and epicatechins.

Anthocyanin biosynthesis is regulated by the MYB-bHLH-WD40 transcription complexes (Jaakola, 2013). Among them, subgroup 5 of MYB transcription factor family is related to proanthocyanidin synthesis, subgroup 7 regulates flavonol biosynthesis, and the subgroup 5 and 6 are regarded as inhibitors and activators of anthocyanin biosynthesis respectively (Dubos et al., 2010). VvMYBA1 and VvMYBA2 were identified as the main transcription factors regulating grape skin coloring, they can bind to cis- elements in the promoter of UFGT and activate its transcription. In white grapes, a retrotransposon gret1 was inserted into the promoter region of VvMYBA1, blocked VvMYBA1 transcription (Kobayashi, 2004), meanwhile VvMYBA2 mutated into a non-functional type, resulting in no anthocyanin accumulation (Azuma et al., 2008). Subgroup III of bHLH have also been reported to be involved in anthocyanin biosynthesis (Zhao et al., 2020; Xi et al., 2021), VvMYC1 interacted with MYBs in grapes and participated in anthocyanin and proanthocyanin biosynthesis regulation (Hichri et al., 2010). Some ERF transcription factors were also found regulating anthocyanin accumulation in Arabidopsis thaliana (Koyama and Sato, 2018), apple (An et al., 2020) and other fruits.

Flavonoids are synthesized in cytoplasm and then transported to vacuoles for storage. At present, the transport mechanism of anthocyanin after synthesis is still unclear. There are four types of anthocyanin transporters reported in grapes: glutathione S-transferase (GST), ATP-binding cassette (ABC) transporters, multidrug and toxic extrusion compound transporters (MATE), and bilitranslocase (BTL) (Petrussa et al., 2013). A total of 161 transporters were identified in the study of grape vacuolar proteome in our laboratory, among which 13 ABC transporters were more abundant at fruit maturity (Kuang et al., 2019).

For most red grape varieties, anthocyanin only exists in vacuoles in the 3-4 layers of cells of skin, and have white flesh and juice, provides the possibility of making white wine from red grapes. Red flesh is very rare among several thousands of grape varieties. Alicante Bouschet is the most well-known red flesh variety, it has rose-red flesh, and is used as a teinturier variety to enhance the color of red wine. Yan73 is a Chinese teinturier variety resulted from a cross between Alicante Bouschet and Muscat Hamburg (Xi et al., 2013). Anthocyanin accumulation happened in the flesh remarkably before veraison, compared with its 
dark-skin and white-flesh parent Muscat Hamburg, the key enzymes of anthocyanin biosynthesis, such as CHS3, UFGT, F3 '5' H, F3H1 and LDOX, and transcription factor MYBA1 were found highly expressed in the flesh of Yan73 (Xie et al., 2015).

Red flesh is a phenotype that exists in many fruits, such as orange, kiwi, apple, plum and others. With higher antioxidant capacity and attractive color, red flesh fruits are welcomed by the market. Our laboratory bred a red-flesh table grape variety 'Mio Red'. Besides fresh consumption, 'Mio Red' is also suitable for making juice. Comparing and analyzing the divergent coloring in flesh and skin of 'Mio Red' berry could provide us further understanding of the coloring regulation of berry flesh, which could benefit breeding of a grape as a healthy snack in the future.

\section{Materials And Methods}

\subsection{Plant material}

'Mio Red' was grown at Shangzhuang experiment station of China Agricultural University, Beijing $\left(40^{\circ} 23 \rrbracket\right.$ $\left.\mathrm{N}, 116^{\circ} 49 \otimes \mathrm{W}\right)$. The anthesis was on May 20, 2020. From June to August, five times sample collection were taken at 20 DAA (Stage I), 35 DAA (end of Stage I), 65 DAA (late Stage II, before veraison), 72 DAA (middle of stage III) and 86 DAA (late stage III) respectively. Three biological replicates were made at each sampling time with 40 berries per replicate. Samples were taken randomly from clusters in different orientations, transported to the laboratory in ice box. The flesh tissues and skin were carefully separated with a scalpel and then quickly frozen in liquid nitrogen. All samples were stored at $-80^{\circ} \mathrm{C}$ for the subsequent analysis.

\subsection{Determination of total anthocyanin}

The method of $\mathrm{Ni}$ et al. (2020b) was used for the determination of total anthocyanin, and some modifications were made. In brief, $0.2 \mathrm{~g}$ grape tissue was ground in liquid nitrogen, then mixed in $1 \mathrm{ml}$ methanol: acetic acid $(99: 1, \mathrm{v} / \mathrm{v})$ solution, and placed in refrigerator at $4{ }^{\circ} \mathrm{C}$ for $24 \mathrm{~h}$. The absorbance of the supernatant was measured by ultraviolet spectrophotometer (Beckman Coulter, Brea, CA, USA) at wavelengths of 530,620 and $650 \mathrm{~nm}$. The relative anthocyanin content was calculated as follows: [(A530-A650)-0.2 × (A650-A620)]/0.1.

\subsection{Determination of total content of flavonoids}

The content of flavonoids was determined as Robinson et al. (2019). After the sample was thoroughly grounded in liquid nitrogen, $0.2 \mathrm{mg}$ was mixed with $1 \mathrm{ml}$ precooled $80 \%$ ethanol, and extracted at $4{ }^{\circ} \mathrm{C}$ for $24 \mathrm{~h}$. After centrifuging at $12,000 \mathrm{rpm}$ at $4{ }^{\circ} \mathrm{C}$ for $20 \mathrm{~min}, 0.5 \mathrm{~mL}$ supernatant was put into a $10 \mathrm{~mL}$ centrifuge tube, then $\left.0.3 \mathrm{~mL} 8 \% \mathrm{NaNO}_{2}, 0.3 \mathrm{~mL} \mathrm{10 \%} \mathrm{Al}_{\left(\mathrm{NO}_{3}\right)}\right)_{3}$ solution, $2 \mathrm{~mL} 2 \mathrm{M} \mathrm{NaOH}$ solution and 4.9 $\mathrm{mL}$ ethanol were added in sequence. After standing for $10 \mathrm{~min}$, the absorbance of the reaction solution was determined at $510 \mathrm{~nm}$. Rutin was used as the standard, and the final flavonoid content was calculated as $\mathrm{mg}$ rutin/g.FW. 


\subsection{Flavonoids metabolome analysis}

About $1 \mathrm{~g}$ of the frozen-stored grape flesh/ skin samples was freezing dried in vacuum, then grounded to powder with a mixer mill (MM 400, Retsch). For each extraction, $100 \mathrm{mg}$ powder was dissolved in $1 \mathrm{ml}$ $70 \%$ methanol, standing at $4{ }^{\circ} \mathrm{C}$ for $24 \mathrm{~h}$, then $10,000 \mathrm{~g}$ centrifuged for $10 \mathrm{~min}$, the supernatant was filtered with a microporous membrane $(0.22 \mu \mathrm{m})$ for LC-MS/MS analysis. Each sample had three biological replicates.

LC-ESI-MS/MS system (HPLC, Shim-pack UFLC SHIMADZU CBM30A system, www.shimadzu.com.cn/; MS, Applied Biosystems 6500 Q TRAP, www.appliedbiosystems.com.cn/) was used. HPLC: column, Waters ACQUITY UPLC HSS T3 C18 $(1.8 \mu \mathrm{m}, 2.1 \mathrm{~mm} * 100 \mathrm{~mm})$; solvent system, water $(0.04 \%$ acetic acid): acetonitrile (0.04\% acetic acid); gradient program, $100: 0 \mathrm{~V} / \mathrm{V}$ at $0 \mathrm{~min}, 5: 95 \mathrm{~V} / \mathrm{V}$ at $11.0 \mathrm{~min}, 5: 95 \mathrm{~V} / \mathrm{V}$ at 12.0min, $95: 5 \mathrm{~V} / \mathrm{V}$ at $12.1 \mathrm{~min}, 95: 5 \mathrm{~V} / \mathrm{V}$ at $15.0 \mathrm{~min}$; flow rate at $0.40 \mathrm{~mL} / \mathrm{min}$; column temperature at 40 ${ }^{\circ} \mathrm{C}$. The injection volume was $2 \mu \mathrm{L}$. The effluent was alternately connected to ESI-triple quadrupole-linear ion trap (Q TRAP)-MS.

Metabolites were identified using Analyst 1.6.3 (AB SCIEX, Ontario, Canada) and quantified by MRM. The metabolites were then submitted to Principal Component Analysis (PCA) and Orthogonal partial leastsquares discrimination analysis (OPLS). Thresholds of VIP (variable impact in project) $\geq 1$ and fold change $\geq 2$ or $\leq 0$. 5 were set as criteria for metabolites with significant differences.

\section{5 transcriptome analysis}

RNA was extracted from berry flesh and skin using a modified CTAB method (Wang et al., 2021) with three biological replicates per sample at each sampling point. RNA quality was checked by $1 \%$ agarose gel electrophoresis, NanoPhotometer spectrophotometer (NanoDrop Technologies, Wilmington, DE, USA), Qubit 2.0 fluorometer (Qubit 2.0, Life Technologies, ThermoFisher Scientific, USA), and Agilent 2100 Bioanalyzer (Agilent Technologies, Palo Alto, CA, USA) before constructing the libraries. The mRNA enrichment and CDNA library establishment were following the standard protocol. The libraries were sequenced by an Illumina HiSeq platform after qualified quality inspection. After removing the sequencing connectors and low-quality reads, the clean reads were annotated against the grape reference genome (Canaguier et al., 2017), the libraries were compared using HISAT2 (Kim, 2015). Fragments per kilobase of exon per million fragments mapped (FPKM) was used to measure the expression level of transcripts. DESEQ2 (Varet et al., 2016) was used to analyze the differential expression among sample groups. The differentially expressed genes were screened with |log2Fold Change| $>=1$ and FDR $<0.05$.

\subsection{Quantitative Real-Time PCR (qRT-PCR)}

According to transcriptome data, 11 structural genes in flavonoid synthesis pathway were selected, and their expression levels at five sampling time points of berry development were validated by qRT-PCR. The primers were listed as Table S1. The PCR reaction was carried out with ABI QuantStudio 6 Q6 (Applied Biosystems) using ChamQ Universal SYBR qPCR Master Mix (Vazyme, China). The $10 \mu \mathrm{L}$ amplification system was consisted of $5 \mu \mathrm{L}$ SYBR qPCR Master Mix, $0.2 \mu \mathrm{L}$ upstream and downstream primer 
respectively $(10 \mu \mathrm{M}), 1 \mu \mathrm{L}$ template, and $3.6 \mu \mathrm{L}$ distilled water. The amplification procedure was $95{ }^{\circ} \mathrm{C} 30$ sec; $95^{\circ} \mathrm{C} 10 \mathrm{sec}, 60{ }^{\circ} \mathrm{C} 30 \mathrm{sec}, 40$ cycles; $95^{\circ} \mathrm{C} 15 \mathrm{sec}, 60^{\circ} \mathrm{C} 60 \mathrm{sec}, 95^{\circ} \mathrm{C} 15 \mathrm{sec}$. All samples were detected by at least three technical repetitions. $\beta$-actin of grapes was used as the reference gene, and the data were quantitatively analyzed by $2^{-\Delta \Delta C T}$ method.

\section{Results}

\subsection{Flavonoids in the skin and flesh of 'Mio Red' at ripening}

The skin and flesh of 'Mio Red' berry both showed coloration at ripening. The skin was uniformly colored, and the anthocyanin in the flesh were mainly concentrated around the style and near the skin (Fig. 1A). Microscopic observation revealed flesh coloration was an independent event other than diffusion of anthocyanin from the skin (Fig. 1B). The anthocyanin content of 'Mio Red' flesh was about 6.2 times lower than that of the skin. The total flavonoids content demonstrated the same trend as anthocyanin, that in the flesh was 14.25 times lower than in the skin (Fig. 1C).

A total of 173 flavonoids were identified in colored skin and flesh of 'Mio Red', which could be further divided into 8 groups, including 63 flavones, 34 flavonols, 18 flavanones, 17 polyphenols, 17 anthocyanins, 16 flavonoids, 5 isoflavones and 3 proanthocyanins (Table S2). Clustering analysis of the flavonoids data revealed obvious differences between and within the two samples (Fig. S1A). Principal component analysis showed a clear trend of separation between the sample groups, PC1 and PC2 contributed $84.23 \%$ and $5.89 \%$ of the differences respectively (Fig. S1B), indicating that there was a significant difference between the metabolites of the skin and the flesh of 'Mio red' grape. Hierarchical heatmap clustered all biological replicates together, and metabolites of the same type together (Fig. S1C), which shows that the results of the flavonoid metabolome were of high reliability.

Sixty-eight metabolites were recruited as of different content between 'Mio Red' flesh and skin by VIP $\geq 1$ and fold change $\geq 2$ or $\leq 0.5$ (Table S3). Compared with the skin, 64 flavonoids were of lower content, and four were of significant higher content in the flesh (Fig. S2A), which were quercetin 3-0-glucoside (flavonol), epicatechin-epiafzelechin (polyphenol), apigenin 6,8-C-diglucoside (flavonoids) and hesperetin 5-0-glucoside (flavanone). Among them, quercetin 3-0-glucoside, epicatechin-epiafzelechin and apigenin 6,8-C-diglucoside were hardly detected in the skin (Table 1). 
Table 1

Differential metabolites in skin and flesh of 'Mio red' grape. The screening conditions were fold change $\geq 2$, fold change $\leq 0.5, \mathrm{VIP} \geq 1$, and can be detected in flesh.

\begin{tabular}{|c|c|c|c|}
\hline \multirow[t]{2}{*}{ Metabolite } & \multicolumn{2}{|l|}{ Content } & \multirow[t]{2}{*}{ LogFC } \\
\hline & Skin & Flesh & \\
\hline \multicolumn{4}{|l|}{ Flavonol } \\
\hline Quercetin 3-O-glucoside & N.D. & $3.70 \mathrm{E}+07$ & 21.97 \\
\hline Kaempferol-3-0-robinoside-7-0-rhamnoside & $3.67 \mathrm{E}+05$ & $1.27 \mathrm{E}+04$ & -4.85 \\
\hline Quercetin & $5.99 \mathrm{E}+06$ & $3.70 \mathrm{E}+04$ & -7.34 \\
\hline Morin & $5.95 \mathrm{E}+06$ & $3.59 E+04$ & -7.37 \\
\hline Myricetin 3-0-galactoside & $2.70 \mathrm{E}+07$ & $1.34 \mathrm{E}+05$ & -7.66 \\
\hline Myricetin & $3.70 \mathrm{E}+07$ & $1.51 \mathrm{E}+05$ & -7.94 \\
\hline Kaempferol 3-0-rhamnoside & $1.06 \mathrm{E}+07$ & $3.91 \mathrm{E}+04$ & -8.08 \\
\hline Quercetin 0-acetylhexoside & $2.93 \mathrm{E}+06$ & $1.06 \mathrm{E}+04$ & -8.1 \\
\hline Quercetin 3-0-rutinoside & $4.93 \mathrm{E}+07$ & $1.59 \mathrm{E}+05$ & -8.28 \\
\hline Quercetin 7-O- $\beta$-D-Glucuronide & $1.30 \mathrm{E}+07$ & $2.61 \mathrm{E}+04$ & -8.96 \\
\hline \multicolumn{4}{|l|}{ Polyphenol } \\
\hline Epicatechin-epiafzelechin & N.D. & $7.64 \mathrm{E}+04$ & 13.05 \\
\hline \multicolumn{4}{|l|}{ Flavone } \\
\hline Apigenin 6,8-C-diglucoside & N.D. & $1.63 \mathrm{E}+04$ & 10.83 \\
\hline Selgin 0-malonylhexoside & $5.20 \mathrm{E}+05$ & $9.67 \mathrm{E}+03$ & -5.75 \\
\hline \multicolumn{4}{|l|}{ Flavanone } \\
\hline Hesperetin 5-0-glucoside & $8.13 \mathrm{E}+05$ & $3.53 E+07$ & 5.44 \\
\hline Naringenin 7-0-neohesperidoside & $3.47 \mathrm{E}+05$ & $1.24 \mathrm{E}+04$ & -4.81 \\
\hline \multicolumn{4}{|l|}{ Flavonoid } \\
\hline Hydroxygenkwanin & $5.68 \mathrm{E}+05$ & $1.10 \mathrm{E}+04$ & -5.69 \\
\hline \multicolumn{4}{|l|}{ Anthocyanins } \\
\hline Delphinidin 3-0-glucoside & $2.38 \mathrm{E}+08$ & $1.51 \mathrm{E}+06$ & -7.3 \\
\hline Petunidin 3, 5-diglucoside & $2.89 \mathrm{E}+07$ & $1.06 \mathrm{E}+05$ & -8.09 \\
\hline
\end{tabular}


Four flavonoid biosynthesis pathways with significant differences were found by locating the differential metabolites in KEGG pathway, among which the flavonoid and flavonol biosynthesis pathway had the largest number of differential metabolites, followed by flavonoid biosynthesis pathway (Fig. S2B). The six common anthocyanins reported in grapes were detected in both the skin and the flesh of 'Mio Red', but their contents were of significant difference in the two tissues. Peonidin derivatives were the main anthocyanin in the skin, while the flesh was dominated by pelargonidin derivatives (Table S4).

\subsection{Transcriptome differences of 'Mio Red' skin and flesh}

Transcriptome sequencing of skin and flesh of ripe 'Mio Red' berries ( 3 biological replicates in each sample), resulted a total of 307,871,952 clean reads, of which 93.8\% reached Q30 (Table S5), $78.01 \%$ $83.80 \%$ of the total clean reads were uniquely matched with grape reference genome (map quality $\geq 30$ ) (Table S6). There were 20,929 genes expressed in both the skin and flesh, 1,326 and 1,531 genes were specifically expressed in the skin and flesh respectively (Fig. 2A).

With $\| \log _{2}$ Fold Change| $>=1$, FDR $<0.05$ and FPKM $\geq 1$ as thresholds, 3,970 DEGs were screened from the two transcriptomes, among which 1,420 genes were up-regulated and 2,550 genes were downregulated in the flesh (Figure S4A). Expression level analysis showed that about $0.2 \%$ of DEGs was of very high expression (FPKM > 1000), 5.4\% were highly expressed (FPKM 100-1000), and $56.2 \%$ had FPKM between 1-100 (Fig. 2B). Further KEGG pathway enrichment revealed that metabolic pathway, flavonoid biosynthesis pathway, phenylpropanoid biosynthesis, flavonoid and flavonol biosynthesis pathway were among the top ranked, in addition to the enrichment of $A B C$ transporter protein genes (Fig. 2C).

\subsection{Expression of structural genes of flavonoid and anthocyanin biosynthesis pathway}

The structural genes which were identified as DEGs in flavonoid biosynthesis included $\mathrm{CHS}, \mathrm{CHI}, \mathrm{F}^{\prime} \mathrm{H}$, $F 3^{\prime} 5^{\prime} H$ and $F 3 H$. The expression of these genes was higher in the skin than the flesh of 'Mio Red' berry. The low expression of $\mathrm{CHS}$ and $\mathrm{CH} /$ in the flesh was in consistent with the low content of total flavonoids in the tissue. Among $F 3 H, F 3^{\prime} H$ and $F 3^{\prime} J^{\prime} H$, $F 3 H$ had the highest FPKM value, followed by $F 3^{\prime} H, F 3^{\prime} 5^{\prime} H$ was with the lowest FPKM, and one $F 3^{\prime} 5^{\prime} H$ (Vitvi06g01895) exhibited the highest fold change in transcript abundance, the FPKM value in the skin was about 12 times higher than that in the flesh. The significant difference in $F 3^{\prime} 5^{\prime} H$ expression could eventually lead to the difference in anthocyanin ratio between delphinidin 3-0-glucoside and petunidin 3, 5-diglucoside (Fig. 3).

Only one DFR gene was found differentially expressed. DFR, acted as the gatekeeper controlling upstream substrate flowing to anthocyanin biosynthesis pathway, the gene was highly expressed in both skin and flesh, specifically in the flesh the FPKM value of DFR was 147. Two ANSDEGs were identified, ANS1 (Vitvi02g00435) and ANS2 (Vitvi11g00565) were found highly expressed in the skin and flesh, respectively. The FPKM value of ANS2 reached 201 in the flesh, which was 5 times of $A N S 2$ expression in 
the skin. Only one DEG was revealed with UFGT, the expression was about 8 times higher in the skin than in the flesh (Fig. 3).

The transcript abundance of genes in flavonol and proanthocyanidin biosynthesis was significantly lower than that of the anthocyanin biosynthesis branch. It is worth noting that the expression level of $F L S$ (Vitvi18g02538) in the flesh was 8.2 times higher than that in the skin, which is in agreement with the metabolome result that the flesh of 'Mio Red' tends to accumulate flavonols compared with the skin. DEGs of $L A R$ and $A N R$, which were found extremely low expression in both the skin and flesh, were in consistent with the detected low proanthocyanidin content.

\subsection{Flavonoid biosynthesis gene expression during berry development}

The expression of flavonoid biosynthesis structure genes was found in well agreement with the specific flavonoid and anthocyanin accumulation character between the flesh and skin. In the phenylpropanoid pathway, $\mathrm{CHS}$ and $\mathrm{CH} /$ demonstrated overall increased expression in the early stage of berry development and decreased from veraison to berry ripening. Overall higher expression was found in the skin than in the flesh, which was in line with the higher flavonoid and anthocyanin content in the skin. The high expression of $\mathrm{CHS}$ and $\mathrm{CH} /$ in the flesh were found at 72 and 65 DAA respectively (Fig. 4).

In the isoflavone pathway, $F 3^{\prime} 5^{\prime} H, F 3^{\prime} H, F 3 H, A N S, U F G T$ and $L A R$ showed overall higher expression in the skin than in the flesh, especially that of UFGT demonstrated the largest expression difference. $F 3^{\prime} H$ and $\mathrm{F} 3 \mathrm{H}$ revealed higher expression in the flesh than in the skin at 86 DAA which supported the late anthocyanin accumulation feature of 'Mio Red' flesh. Moreover, the expression of FLS and ANR, key genes for the biosynthesis of flavonols and proanthocyanins, had generally higher expression in flesh than the skin (Fig. 4), supported the assayed high apigenin 6,8-C-diglucoside, epicatechin-epiafzelechin, hesperetin 5-0-glucoside and quercetin 3-0-glucoside content found in the flesh.

\subsection{Identification and correlation analysis of transcription factors related to flavonoid biosynthesis}

The important transcription factor family associated with fruit growth and development identified in the transcription of skin and flesh of 'Mio Red' included 45 AP2/ERF, 39 MYB (including MYB-related), 32 bHLH, 27 WRKY, 23 NAC and 3 WD40. Most of the of NAC and WD40 were up-regulated in the flesh. The number of up-regulated and down-regulated genes of bHLH was the same. Members of the rest transcription factor families were mostly down-regulated in the flesh (Fig. 5A).

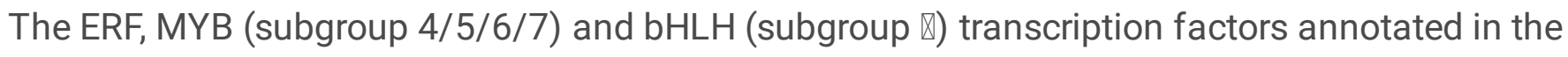
transcriptome of 'Mio Red' related to the biosynthesis of anthocyanin were separately constructed into phylogenetic trees to screen TFs that might be involved in berry flesh coloration regulation. A total of 12 ERF, 9 MYB and 3 bHLH genes were screened. ERF Vitvi18g01617, ERFBP-like1, Vitvi18g02398 were 
highly expressed in both skin and flesh. They were clustered with PbERF3 of pear, JcERF035 of physic nut (Jatropha curcas L.) and MdERF38 of apple, respectively (Fig. 5B).

Among the MYB transcription factors, MYBA1/2/3 clustered in subgroup 6, and was highly expressed in both skin and flesh, which may directly regulate fruit coloring. MYBC2-L3 and MYB3 were clustered in subgroup 4, which may play the role of feedback regulation and inhibit the excessive accumulation of anthocyanin. MYBPA7 was clustered in subgroup 5, its expression level was low, which was consistent with the low content of proanthocyanin in 'Mio Red' fruit. MYBPA1 was clustered in subgroup 7, homologous to VvMYBPA1 and VvMYBF1 (Fig. 5C). Among bHLH transcription factors, MYC2 with high expression level and cluster with AtMYC2/4/5 of Arabidopsis thaliana. bHLH93 and bHLH116, which were clustered together with AtbHLH33, were highly expressed in the skin and flesh, respectively (Fig. 5D).

There were only 3 differentially expressed WD40 found in 'Mio Red', and their expression level was low. NAC and bZIP TFs family were screened with FPKM $\geq 10$ in the skin or flesh, 6 and 2 transcription factors were obtained respectively. Three NACs namely NAC100, NAC71 and NAC83, and 2 bZIPs i.e. BZIP41 and BZIP9, were significantly upregulated in the flesh than the skin (Fig. 6A).

MBW complex mainly regulates the biosynthesis of anthocyanins. The correlation analysis of MYB, bHLH and WD40 transcription factors showed that the activator MYBA1/2/3 (subgroup 6) and the inhibitor MYB3/C2-L1/C2-L3 (subgroup 4) were positively correlated with bHLH93. Three WD40 genes were negatively correlated with all MYB transcription factors except MYB167, and positively correlated with bHLH116 and MYC2 (Fig. 6B).

\subsection{Integrated analysis of DEG and DAM related to flavonoid biosynthesis and transport}

The genes and metabolites with high abundance in flavonoid pathway and Pearson correlation coefficient greater than 0.8 were selected for correlation analysis. In Figure 7A, genes and metabolites were negatively correlated in quadrants 1 and 9 , positively correlated in quadrants 3 and 7 . The results showed that the number of genes and metabolites in quadrants 7 and 9 was much higher than that in quadrants 3 and 1 , which indicated that the genes and metabolites related to flavonoids in the flesh were significantly down-regulated compared with the skin, regardless whether genes were positively or negatively regulated. Combined DEGs and DAMs KEGG enrichment analysis showed that only flavonoid biosynthesis pathway was significantly enriched $(p<0.05)$ (Fig. 7B).

The correlation network was constructed with genes and metabolites enriched in flavonoids, flavone and flavonol, and anthocyanin biosynthesis, the coefficient of correlation was higher than 0.8. Flavonoids, flavone and flavonol biosynthesis were associated with most genes. Three UFGT genes were screened correlating with anthocyanin biosynthesis, which could play a key role in the differential coloring of flesh and skin (Fig. S3). 
In this study, 23 putative flavonoid-related transporters were identified in 3 types: 15 GST, 5 MATE and 3 $A B C$ transporter. They may implement the transporting of anthocyanins, proanthocyanins and flavonols. The results of transcriptome showed that all the transporter genes were higher expressed in the skin than the flesh. Among the transporters GST14 and MATE4 were of high FPKM in both the skin and flesh. Correlation analysis with metabolites showed that most transporters were positively correlated with DAMs. Interestingly, GST8 transporter was negatively correlated with most flavonoids including two anthocyanins: delphinidin 3-0-glucoside, petunidin 3, 5-diglucoside, but only positively correlated with quercetin 3-0-glucoside (flavonol), hesperetin 5-0-glucoside (flavanone) and epicatechin-epiafzelechin (polyphenol) (Fig. 7C).

\section{Discussion}

\subsection{Pattern of anthocyanin accumulation in the flesh of different red flesh grape varieties}

Most of the colored grapes only accumulate anthocyanin in the skin at berry ripening, veraison is the common start point of skin anthocyanin accumulation. However, flesh anthocyanin development time is of remarkable diversity among the small population of red flesh varieties. The flesh coloring of the teinturier wine variety Yan73 occurred in the stage II of the double sigmoid curve and was obviously earlier than its skin coloring (He et al., 2010). 'Summer Black' is a table grape variety with a V. vinifera and $V$. labrusca hybrid background. The flesh and skin of 'Summer Black' red-flesh mutant start coloring almost at the same time at berry ripening (Zhang et al., 2018b). In the case of 'Mio red', the coloration of the flesh was significantly later than that of the skins. Since flesh coloration occurs only when the skin was dark, the coloration of 'Mio Red' flesh may have a non-light-dependent character.

There was small difference in anthocyanin content between the skin and flesh of Yan73, and the proportion of different anthocyanin derivatives was similar, with the highest content of malvidin and peonidin derivatives and the lowest content of pelargonidin (Chen et al., 2018). The anthocyanin profile in the flesh and skin of 'Summer Black' red flesh mutant was also similar, the appearance of the red flesh trait may relate to the enhanced anthocyanin biosynthesis in the whole berry. As the color of the skin gradually increasing, the shading effect prevents the synthesis of anthocyanin in the flesh, which resulted the anthocyanin level of the flesh far lower than that of the skin (Zhang et al., 2018b). DAMs in the skin and flesh of 'Mio red' grape include flavonols, flavonoids and anthocyanins, more than $1 / 3$ of flavonoids metabolites were of significant content different, which implied that the main flavonoids synthesized in the skin and flesh of 'Mio red' grape were different.

It was previously thought that grapes did not contain pelargonidin (Jeong et al., 2006), as the DFR of the studied varieties could not effectively reduce dihydrokaempferol to produce leucopelargonidin (Xie et al., 2004). Later on, pelargonidin was detected with extremely low content in $V$. vinifera varieties such as Cabernet Sauvignon and Pinot Noir. In Yan73 grape, DFR can complete the reduction of dihydrokaempferol, but it was more inclined to synthesize cyanidin and delphinin using dihydroquercetin 
and dihydromyricetin as substrates, so the content of pelargonidin derivatives in Yan73 was low (Xie et al., 2018). However, in 'Mio Red' grape, especially in the flesh, pelargonidin accumulated to a certain extent.

Previous studies reported that the expression pattern of ANS varies within different grape varieties, ANS protein accumulates in various tissues of grapevine and exhibits different effects on secondary metabolites (Boss et al., 1996). In grape leaves, ANS was mainly involved in the biosynthesis of PAs, while in stem phloem, ANS responds to both anthocyanin and PAs (Wang et al., 2011). There were two main ANS in 'Mio Red' berry, ANS1 (Vitvi11g00565) and ANS2 (Vitvi02g00435), which were highly expressed in the skin and flesh, respectively. The specific spatiotemporal expression of the two ANS genes could be involved in the divergent coloration initiation and anthocyanin content in the skin and flesh of 'Mio Red' grape.

\subsection{Transcription factors on flavonoid accumulation}

At present, there are few studies on transcription factors that regulate the expression of structural genes that synthesize flavonoids in grape flesh. The study of Yan73 suggested that the accumulation of anthocyanin in flesh tissue was probably due to specific expression of the structural genes and TFs, which was coordinated and regulated by VvMYBA1 transcription activator and VvMYBC2-L1 transcription repressor (Xie et al., 2019). Comparing the metabolic and transcriptomic profiles of the skin and flesh of three table grape varieties ('Kyoho', 'Wink', 'Italia'), it was found that besides anthocyanin, flavonol and flavanol were also different in a wide range, and MYB24, MADS5 and two ubiquitin proteins (RHA2) were suggested as promising candidates for regulating flavonoids biosynthesis in grape (Lu et al., 2021).

In the present study, TFs related to flavonoid biosynthesis were screened, and AP2/ERF took a high number. The direct relationship between ERFs and flavonoid biosynthesis is still unclear. ERFs generally regulated anthocyanin accumulation by interacting with MYB or bHLH TFs. It was reported that in apple MdERF1B and MdERF3 promote anthocyanin and proanthocyanidin biosynthesis by interacting with MdMYBs (Zhang et al., 2018a; An et al., 2018). In pear, PpERF105 inhibits anthocyanin biosynthesis by inducing the expression of a transcription repressor PpMYB140 (Ni et al., 2019).

Among of the 11 MYBs screened from our transcriptome and phylogenetic tree analysis, the expression of MYBA1, MYBA2 and MYBA3 as activators in the skin was significantly higher than that in the flesh. MYBA1 and MYBA2 regulate anthocyanin biosynthesis by binding to the promoter of UFGT (Walker et al., 2007). As a homolog of MYBA1/A2, MYBA3 was also considered to play an activator role in anthocyanin biosynthesis (Leng et al., 2020). The expression patterns of these three MYBs in the flesh of 'Mio Red' could be important to the flesh coloration. MYBPA7, VvMYBPA2 and VvMYBPAR regulating proanthocyanin biosynthesis were clustered into subgroup 5 (Koyama et al., 2014). The low expression of MYBPA7, especially in the flesh, could be the main reason for the low accumulation of proanthocyanins in 'Mio Red'. MYBPA1 and its homologous proteins VvMYBPA1, VvMYBF1 and AtMYB12 in subgroup 7 regulate flavonol biosynthesis (Czemmel et al., 2009). Studies have shown that VvMYBPA1 activates 
LAR, ANR and promoters of several flavonoid pathway structural genes including CHS, F3' 5 'H and ANS, participates the biosynthesis of procyanidins (Bogs et al., 2007).

The existence of suppressors is essential, which can fine-tune the synthesis process when anthocyanin could accumulate too much, and maintain a homeostasis of fruits (LaFountain and Yuan, 2021). MYB inhibitors are generally divided into two types, MYB4 and MYBC2-L1 homologs. MYB4 inhibited small molecular weight phenolic compounds, while MYBC2-L1/L3 regulated the level of flavonoids and balanced the action of activators (Cavallini et al., 2015). MYBC2-L1 and MYB3 were screened in this study as inhibitors, which could inhibit the accumulation of flavonoids. In the correlation analysis of MBW complex in 'Mio Red', both MYB activators and inhibitors were positively correlated with VvbHLH93.

Among the $3 \mathrm{bHLH}$ screened from the phylogenetic tree, bHLH 116 and bHLH93 were clustered together with AtbHLH116 (ICE1) and AtbHLH93 (ICE2), they were reported a role in low temperature stress (Fursova et al., 2009). MYC2 clustered with AtMYC2, AtbHLH3. In Arabidopsis thaliana, MYC2 promotes flavonoid biosynthesis in the downstream of JA signaling by regulating the expression of positive regulators PAP1 and EGL3. MYC2 can also regulate anthocyanin biosynthesis in corn (Zea mays) (Dombrecht et al., 2007). MYC2 had a high expression in the skin and flesh of 'Mio Red', which could promote the coloring.

NAC and BZIP family TFs participate in various life processes in plants, including the regulation of flavonoid biosynthesis. Overexpression of PaNACO3 in spruce reduced the accumulation of flavonoids (Dalman et al., 2017). Overexpression of VvibZIP22 in grapes significantly increased the content of flavonoids (Malacarne et al., 2016). These two kinds of TFs may also contribute to the synthesis of flavonoids in 'Mio Red' grape.

\subsection{Other regulatory factors on flavonoid accumulation}

Flavonoids are synthesized in the cytoplasm and then transported to vacuoles for storage or to other destinations where they function as bioactive molecules. Different compartment of flavonoids biosynthesis, storage and function requires effective transport mechanisms to realize their biological functions. In grapes, VvABCC1, VvGST4, AM1 and AM3 were confirmed roles in anthocyanin transport, and VvGST1 in proanthocyanidin transportation (Zhao, 2015). VvMATE1/2, which are homologs to AtTT12 in Arabidopsis thaliana, was located in vacuoles and Golgi complex respectively (Perez-Diaz et al., 2014). Stress-induced TaGSTL1 selectively recognized flavonol in wheat (Dixon and Edwards, 2010). Our DEG and DAM integrated analysis suggested GST14 and MATE4 may play a role in the transport of flavonoids, while GST8 could involve in the transportation of quercetin 3-0-glucoside, hesperetin 5-0glucoside and epicatechin-epiafzelechin. Compared with the skin, the expression of transporter genes in the flesh was lower, the inefficient transport after synthesis could be another reason for the less accumulation of flavonol in the flesh.

Structural genes and regulatory genes in flavonoid biosynthesis pathway can be induced by light (Jaakola et al., 2010). Light-induced anthocyanin accumulation requires light-responsive elements, 
including Constitutively Photomorphogenic 1 (COP1) and Long Hypocotyl 5 (HY5) (Maier et al., 2013). In apples, MdMYB1 accumulated in light and degraded through an ubiquitin-dependent pathway in dark (Li et al., 2012). In addition, under low ultraviolet irradiation, the transcription levels of FLS, HY5 and MYB10 in apple fruits were down-regulated, and anthocyanin and flavonol content decreased (Henry-Kirk et al., 2018). PpBBX16, an activator of light-induced anthocyanin accumulation, was found in pears (Bai et al., 2019). In nature, there are many red flesh fruits whose flesh coloration does not depend on light signals and in a different time line to their light dependent coloration skin, such as fig (Ficus carica L.) (Cui et al., 2021). Anthocyanin accumulation in the flesh of 'Mio Red' berry happens in a low light if not a light absent condition, elucidation of the mechanism could help us to improve the coloring of the historical fruit.

\section{Conclusion}

'Mio Red', a dark skin table grape develops color in the flesh at ripening, the color was identified as anthocyanin accumulation. The flesh color development pattern is of remarkable difference from the other reported red flesh grape variety. Metabolome analysis revealed peonidin and pelargonidin derivatives as the leading anthocyanin in 'Mio Red' skin and flesh respectively, exhibited bio-diversity in the trait of red flesh formation. Transcriptome analysis uncovered that most of the catalyze enzymes in the anthocyanin biosynthesis pathway was coded by two genes, that showed different expression preference in the flesh and skin. A set of transcription factors were co-expression analyzed and recruited for the future function validation. The results further our understanding of flavonoids and anthocyanin accumulation in grape flesh, and provide new clues for subsequent regulatory mechanism study. Moreover, this study could pave the way for breeding of future red-flash seedless table grapes having the health benefit of red wines.

\section{Declarations}

\section{Funding}

The work was supported by 111 Project (B17043).

\section{Data availability}

The raw RNA-seq data are available at: https://www.ncbi.nlm.nih.gov/bioproject/PRJNA781680.

\section{Declarations}

There is no conflict of interest.

\section{Author Contributions}

$\mathrm{HM}$ and MS designed experiments, RL and MS prepared samples for RNA-seq, RL completed physiological experiments and qRT-PCR, RL and MS completed all data collation and analysis, RL, MS 
and $\mathrm{HM}, \mathrm{ZW}, \mathrm{YZ}, \mathrm{CH}, \mathrm{AP}$ wrote articles and made revisions. All authors have read and approved the manuscript for publication.

\section{References}

Agati, G., Azzarello, E., Pollastri, S., \& Tattini, M. (2012). Flavonoids as antioxidants in plants: location and functional significance. Plant Science, 196, 67-76. https://doi.org/10.1016/j.plantsci.2012.07.014

Ageorges, A., Fernandez, L., Vialet, S., Merdinoglu, D., Terrier, N., and Romieu, C. (2006). Four specific isogenes of the anthocyanin metabolic pathway are systematically co-expressed with the red colour of grape berries. Plant Science 170(2), 372-383. doi: 10.1016/j.plantsci.2005.09.007.

Allan, A.C., and Espley, R.V. (2018). MYBs Drive Novel Consumer Traits in Fruits and Vegetables. Trends Plant Sci 23(8), 693-705. doi: 10.1016/j.tplants.2018.06.001.

An, J.P., Wang, X.F., Li, Y.Y., Song, L.Q., Zhao, L.L., You, C.X., et al. (2018). EIN3-LIKE1, MYB1, and ETHYLENE RESPONSE FACTOR3 Act in a Regulatory Loop That Synergistically Modulates Ethylene Biosynthesis and Anthocyanin Accumulation. Plant Physiology 178(2), 808-823. doi: 10.1104/pp.18.00068.

An, J.P., Zhang, X.W., Bi, S.Q., You, C.X., Wang, X.F., and Hao, Y.J. (2020). The ERF transcription factor MdERF38 promotes drought stress-induced anthocyanin biosynthesis in apple. Plant $\mathrm{J}$ 101(3), 573-589. doi: $10.1111 /$ tpj. 14555 .

Azuma, A., Kobayashi, S., Mitani, N., Shiraishi, M., Yamada, M., Ueno, T., et al. (2008). Genomic and genetic analysis of Myb-related genes that regulate anthocyanin biosynthesis in grape berry skin. Theor Appl Genet 117(6), 1009-1019. doi: 10.1007/s00122-008-0840-1.

Bai, S., Tao, R., Tang, Y., Yin, L., Ma, Y., Ni, J., et al. (2019). BBX16, a B-box protein, positively regulates light-induced anthocyanin accumulation by activating MYB10 in red pear. Plant Biotechnol J 17(10), 1985-19 97. doi: 10.1111/pbi.13114.

Bogs, J., Jaffe, F.W., Takos, A.M., Walker, A.R., and Robinson, S.P. (2007). The grapevine transcription factor VvMYBPA1 regulates proanthocyanidin synthesis during fruit development. Plant Physiol 143(3), 1347-1361. doi: 10.1104/pp.106.093203.

Boss, P.K., Davies, C., and Robinson, S.P. (1996). Expression of anthocyanin biosynthesis pathway genes in red and white grapes. Plant Molecular Biology 32(3), 565-569. doi: 10.1007/bf00019111.

Canaguier, A., Grimplet, J., Di Gaspero, G., Scalabrin, S., Duchene, E., Choisne, N., et al. (2017). A new version of the grapevine reference genome assembly (12X.v2) and of its annotation (VCost.v3). Genom Data 14, 56-62. doi: 10.1016/j.gdata.2017.09.002. 
Cavallini, E., Matus, J.T., Finezzo, L., Zenoni, S., Loyola, R., Guzzo, F., et al. (2015). The phenylpropanoid pathway is controlled at different branches by a set of R2R3-MYB C2 repressors in grapevine. Plant Physiol 167(4), 1448-1470. doi: 10.1104/pp.114.256172.

Chen, W.K., Wang, Y., Gao, X.T., Yang, X.H., He, F., Duan, C.Q., et al. (2018). Flavonoid and aromatic profiles of two Vitis vinifera L. teinturier grape cultivars. Australian Journal of Grape and Wine Research 24(3), 379-389. doi: 10.1111/ajgw.12336.

Coombe, B. G., \& Bishop, G. R. (1980). Development of the grape berry. II. Changes in diameter and deformability during veraison. Australian Journal of Agricultural Research, 31(3), 499-509. https://doi.org/10.1071/AR9800499

Cui, Y., Zhai, Y., Flaishman, M., Li, J., Chen, S., Zheng, C., et al. (2021). Ethephon induces coordinated ripening acceleration and divergent coloration responses in fig (Ficus carica L.) flowers and receptacles. Plant Mol Biol 105(4-5), 347-364. doi: 10.1007/s11103-020-01092-x.

Czemmel, S., Stracke, R., Weisshaar, B., Cordon, N., Harris, N.N., Walker, A.R., et al. (2009). The grapevine R2R3-MYB transcription factor VvMYBF1 regulates flavonol synthesis in developing grape berries. Plant Physiol 151(3), 1513-1530. doi: 10.1104/pp.109.142059.

Dalman, K., Wind, J.J., Nemesio-Gorriz, M., Hammerbacher, A., Lunden, K., Ezcurra, I., et al. (2017). Overexpression of PaNAC03, a stress induced NAC gene family transcription factor in Norway spruce leads to reduced flavonol biosynthesis and aberrant embryo development. BMC Plant Biol 17(1), 6. doi: 10.1186/s12870-016-0952-8.

Dixon, D.P., and Edwards, R. (2010). Roles for stress-inducible lambda glutathione transferases in flavonoid metabolism in plants as identified by ligand fishing. J Biol Chem 285(47), 36322-36329. doi: 10.1074/jbc.M110.164806.

Dombrecht, B., Xue, G.P., Sprague, S.J., Kirkegaard, J.A., Ross, J.J., Reid, J.B., et al. (2007). MYC2 differentially modulates diverse jasmonate-dependent functions in Arabidopsis. Plant Cell 19(7), 22252245. doi: 10.1105/tpc.106.048017.

Dubos, C., Stracke, R., Grotewold, E., Weisshaar, B., Martin, C., and Lepiniec, L. (2010). MYB transcription factors in Arabidopsis. Trends Plant Sci 15(10), 573-581. doi: 10.1016/j.tplants.2010.06.005.

Fuleki, T., and Ricardo-da-Silva, J.M. (1997). Catechin and procyanidin composition of seeds from grape cultivars grown in Ontario. Journal of Agricultural and Food Chemistry 45(4), 1156-1160. doi: 10.1021/jf960493k.

Fursova, O.V., Pogorelko, G.V., and Tarasov, V.A. (2009). Identification of ICE2, a gene involved in cold acclimation which determines freezing tolerance in Arabidopsis thaliana. Gene 429(1-2), 98-103. doi: 10.1016/j.gene.2008.10.016. 
He, J.J., Liu, Y.X., Pan, Q.H., Cui, X.Y., and Duan, C.Q. (2010). Different anthocyanin profiles of the skin and the pulp of Yan7 (Muscat Hamburg x Alicante Bouschet) grape berries. Molecules 15(3), 1141-1153. doi: 10.3390/molecules15031141.

Henry-Kirk, R.A., Plunkett, B., Hall, M., McGhie, T., Allan, A.C., Wargent, J.J., et al. (2018). Solar UV light regulates flavonoid metabolism in apple (Malus x domestica). Plant Cell Environ 41(3), 675-688. doi: $10.1111 /$ pce. 13125 .

Hichri, I., Heppel, S.C., Pillet, J., Leon, C., Czemmel, S., Delrot, S., et al. (2010). The basic helix-loop-helix transcription factor MYC1 is involved in the regulation of the flavonoid biosynthesis pathway in grapevine. Mol Plant 3(3), 509-523. doi: 10.1093/mp/ssp118.

Jaakola, L. (2013). New insights into the regulation of anthocyanin biosynthesis in fruits. Trends in Plant Science 18(9): 477-483.

Jaakola, L., Poole, M., Jones, M.O., Kamarainen-Karppinen, T., Koskimaki, J.J., Hohtola, A., et al. (2010). A SQUAMOSA MADS box gene involved in the regulation of anthocyanin accumulation in bilberry fruits. Plant Physiol 153(4), 1619-1629. doi: 10.1104/pp.110.158279.

Jeong, S.T., Goto-Yamamoto, N., Hashizume, K., and Esaka, M. (2006). Expression of the flavonoid 3'hydroxylase and flavonoid 3',5'-hydroxylase genes and flavonoid composition in grape (Vitis vinifera). Plant Science 170(1), 61-69. doi: 10.1016/j.plantsci.2005.07.025.

Jiu, S., Guan, L., Leng, X., Zhang, K., Haider, M.S., Yu, X., et al. (2021). The role of VvMYBA2r and VvMYBA2 $w$ alleles of the MYBA2 locus in the regulation of anthocyanin biosynthesis for molecular breeding of grape (Vitis spp.) skin coloration. Plant Biotechnol J. doi: 10.1111/pbi.13543.

Kim, D., Langmead, B., and Salzberg, S.L. (2015). HISAT: a fast spliced aligner with low memory requirements. Nature Methods 12(4), 357-U121. doi: 10.1038/nmeth.3317.

Kobayashi, S. (2004). Retrotransposon-Induced Mutations in Grape Skin Color. Science 304(5673), 982982. doi: 10.1126/science. 1095011.

Koyama, K., Numata, M., Nakajima, I., Goto-Yamamoto, N., Matsumura, H., and Tanaka, N. (2014). Functional characterization of a new grapevine MYB transcription factor and regulation of proanthocyanidin biosynthesis in grapes. J Exp Bot 65(15), 4433-4449. doi: 10.1093/jxb/eru213.

Koyama, T., and Sato, F. (2018). The function of ETHYLENE RESPONSE FACTOR genes in the lightinduced anthocyanin production of Arabidopsis thaliana leaves. Plant Biotechnology 35(1), 87-91. doi: 10.5511/plantbiotechnology.18.0122b.

Kuang, L., Chen, S., Guo, Y., and Ma, H. (2019). Quantitative Proteome Analysis Reveals Changes in the Protein Landscape During Grape Berry Development With a Focus on Vacuolar Transport Proteins. Front Plant Sci 10, 641. doi: 10.3389/fpls.2019.00641. 
LaFountain, A.M., and Yuan, Y.W. (2021). Repressors of anthocyanin biosynthesis. New Phytol 231(3), 933-949. doi: 10.1111/nph.17397.

Leng, F., Cao, J., Ge, Z., Wang, Y., Zhao, C., Wang, S., et al. (2020). Transcriptomic Analysis of Root Restriction Effects on Phenolic Metabolites during Grape Berry Development and Ripening. J Agric Food Chem 68(34), 9090-9099. doi: 10.1021/acs.jafc.0c02488.

Li, Y.Y., Mao, K., Zhao, C., Zhao, X.Y., Zhang, H.L., Shu, H.R., et al. (2012). MdCOP1 ubiquitin E3 ligases interact with MdMYB1 to regulate light-induced anthocyanin biosynthesis and red fruit coloration in apple. Plant Physiol 160(2), 1011-1022. doi: 10.1104/pp.112.199703.

Lu, S., Wang, J., Zhuge, Y., Zhang, M., Liu, C., Jia, H., et al. (2021). Integrative Analyses of Metabolomes and Transcriptomes Provide Insights into Flavonoid Variation in Grape Berries. J Agric Food Chem. doi: 10.1021/acs.jafc. 1 c02703.

Maier, A., Schrader, A., Kokkelink, L., Falke, C., Welter, B., Iniesto, E., et al. (2013). Light and the E3 ubiquitin ligase COP1/SPA control the protein stability of the MYB transcription factors PAP1 and PAP2 involved in anthocyanin accumulation in Arabidopsis. Plant J 74(4), 638-651. doi: 10.1111/tpj.12153.

Malacarne, G., Coller, E., Czemmel, S., Vrhovsek, U., Engelen, K., Goremykin, V., et al. (2016). The grapevine VvibZIPC22 transcription factor is involved in the regulation of flavonoid biosynthesis. J Exp Bot 67(11), 3509-3522. doi: 10.1093/jxb/erw181.

Ni, J., Premathilake, A.T., Gao, Y., Yu, W., Tao, R., Teng, Y., et al. (2020a). Ethylene-activated PpERF105 induces the expression of the repressor-type R2R3-MYB gene PpMYB140 to inhibit anthocyanin biosynthesis in red pear fruit. Plant J. doi: 10.1111/tpj.15049.

Ni, J., Zhao, Y., Tao, R., Yin, L., Gao, L., Strid, A., et al. (2020b). Ethylene mediates the branching of the jasmonate-induced flavonoid biosynthesis pathway by suppressing anthocyanin biosynthesis in red Chinese pear fruits. Plant Biotechnol J 18(5), 1223-1240. doi: 10.1111/pbi.13287.

Perez-Diaz, R., Ryngajllo, M., Perez-Diaz, J., Pena-Cortes, H., Casaretto, J.A., Gonzalez-Villanueva, E., et al. (2014). VvMATE1 and VvMATE2 encode putative proanthocyanidin transporters expressed during berry development in Vitis vinifera L. Plant Cell Rep 33(7), 1147-1159. doi: 10.1007/s00299-014-1604-9.

Petrussa, E., Braidot, E., Zancani, M., Peresson, C., Bertolini, A., Patui, S., et al. (2013). Plant flavonoidsbiosynthesis, transport and involvement in stress responses. Int J Mol Sci 14(7), 14950-14973. doi: 10.3390/ijms140714950.

Robinson, S.P., Pezhmanmehr, M., Speirs, J., McDavid, D.A.J., Hooper, L.C., Rinaldo, A.R., et al. (2019). Grape and wine flavonoid composition in transgenic grapevines with altered expression of flavonoid hydroxylase genes. Australian Journal of Grape and Wine Research 25(3), 293-306. doi:

10.1111/ajgw.12393. 
Varet, H., Brillet-Gueguen, L., Coppee, J.-Y., and Dillies, M.-A. (2016). SARTools: A DESeq2-and EdgeRBased R Pipeline for Comprehensive Differential Analysis of RNA-Seq Data. Plos One 11(6). doi: 10.1371/journal.pone.0157022.

Walker, A.R., Lee, E., Bogs, J., McDavid, D.A., Thomas, M.R., and Robinson, S.P. (2007). White grapes arose through the mutation of two similar and adjacent regulatory genes. Plant J 49(5), 772-785. doi: 10.1111/j.1365-313X.2006.02997.x.

Wang, H., Wang, W., Li, H., Zhang, P., Zhan, J., and Huang, W. (2011). Expression and tissue and subcellular localization of anthocyanidin synthase (ANS) in grapevine. Protoplasma 248(2), 267-279. doi: 10.1007/s00709-010-0160-6.

Wang, Z., Song, M., Wang, Z., Chen, S., and Ma, H. (2021). Metabolome and transcriptome analysis of flavor components and flavonoid biosynthesis in fig female flower tissues (Ficus carica L.) after bagging. BMC Plant Biol 21(1), 396. doi: 10.1186/s12870-021-03169-1.

$\mathrm{Xi}, \mathrm{H} ., \mathrm{He}, \mathrm{Y}$, and Chen, H. (2021). Functional Characterization of SmbHLH13 in Anthocyanin Biosynthesis and Flowering in Eggplant. Horticultural Plant Journal 7(1), 73-80. doi: 10.1016/j.hpj.2020.08.006.

Xi, Z.M., Meng, J.F., Huo, S.S., Luan, L.Y., Ma, L.N., and Zhang, Z.W. (2013). Exogenously applied abscisic acid to Yan73 (V. vinifera) grapes enhances phenolic content and antioxidant capacity of its wine. Int $J$ Food Sci Nutr 64(4), 444-451. doi: 10.3109/09637486.2012.746291.

Xie, D.Y., Jackson, L.A., Cooper, J.D., Ferreira, D., and Paiva, N.L. (2004). Molecular and biochemical analysis of two cDNA clones encoding dihydroflavonol-4-reductase from medicago truncatula. Plant Physiology 134(3), 979-994. doi: 10.1104/pp.103.030221.

Xie, S., Qiao, X., Chen, H., Nan, H., and Zhang, Z. (2019). Coordinated Regulation of Grape Berry Flesh Color by Transcriptional Activators and Repressors. J Agric Food Chem 67(42), 11815-11824. doi: 10.1021/acs.jafc.9b05234.

Xie, S., Song, C., Wang, X., Liu, M., Zhang, Z., and Xi, Z. (2015). Tissue-Specific Expression Analysis of Anthocyanin Biosynthetic Genes in White- and Red-Fleshed Grape Cultivars. Molecules 20(12), 2276722780. doi: 10.3390/molecules201219883.

Xie, S., Zhao, T., Zhang, Z., and Meng, J. (2018). Reduction of Dihydrokaempferol by Vitis vinfera Dihydroflavonol 4-Reductase to Produce Orange Pelargonidin-Type Anthocyanins. J Agric Food Chem 66(13), 3524-3532. doi: 10.1021/acs.jafc.7b05766.

Yang, B., Wei, Y., Liang, C., Guo, J., Niu, T., Zhang, P., et al. (2021). VvANR silencing promotes expression of VvANS and accumulation of anthocyanin in grape berries. Protoplasma. doi: 10.1007/s00709-02101698-y. 
Zhang, J., Xu, H., Wang, N., Jiang, S., Fang, H., Zhang, Z., et al. (2018a). The ethylene response factor MdERF1B regulates anthocyanin and proanthocyanidin biosynthesis in apple. Plant Molecular Biology 98(3), 205-218. doi: 10.1007/s11103-018-0770-5.

Zhang K, Liu Z, Guan L, et al., 2018. Changes of Anthocyanin Component Biosynthesis in 'Summer Black' Grape Berries after the Red Flesh Mutation Occurred. J Agric Food Chem 66, 9209-18.

Zhao, J. (2015). Flavonoid transport mechanisms: how to go, and with whom. Trends Plant Sci 20(9), 576-585. doi: 10.1016/j.tplants.2015.06.007.

Zhao, R., Song, X., Yang, N., Chen, L., Xiang, L., Liu, X.Q., et al. (2020). Expression of the subgroup IIIf bHLH transcription factor CpbHLH1 from Chimonanthus praecox (L.) in transgenic model plants inhibits anthocyanin accumulation. Plant Cell Rep 39(7), 891-907. doi: 10.1007/s00299-020-02537-9.

\section{Figures}

A

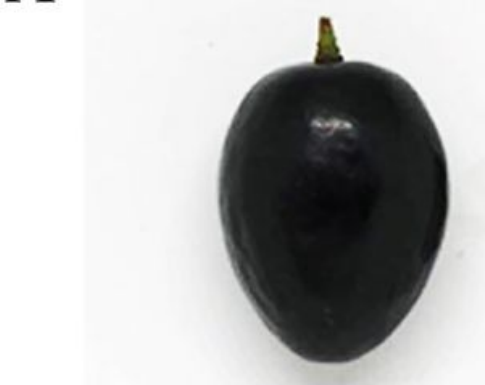

B

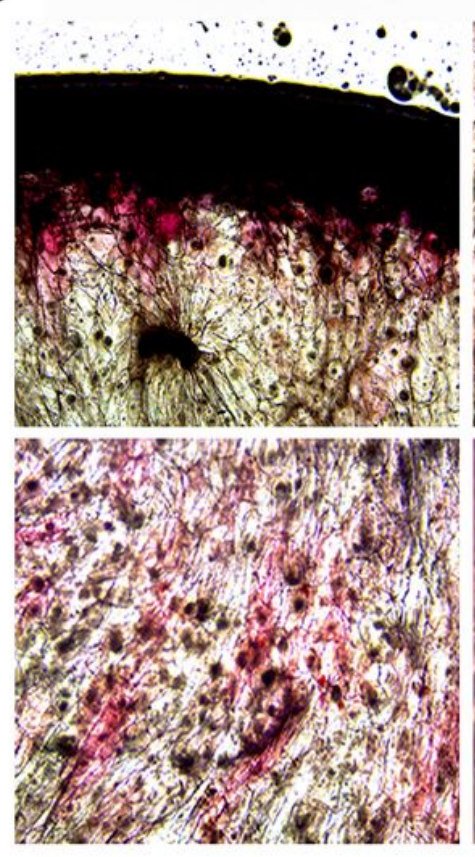

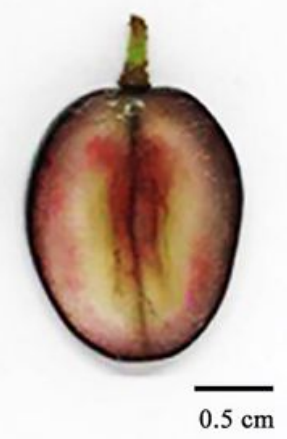
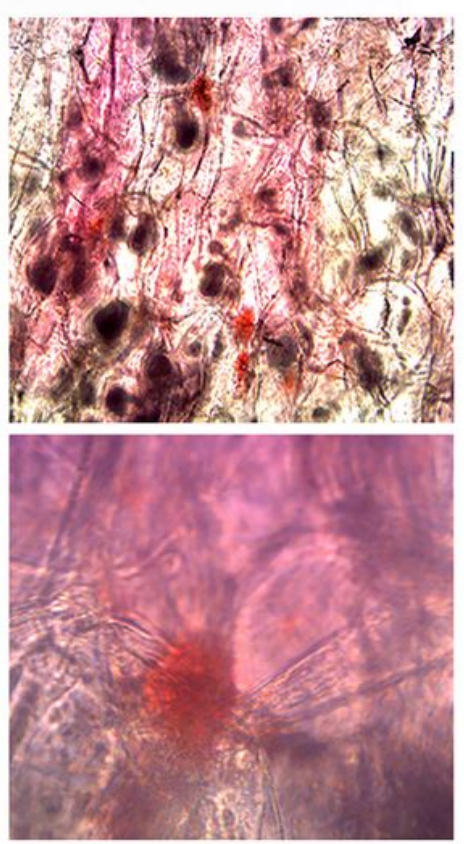

$\mathrm{C}$
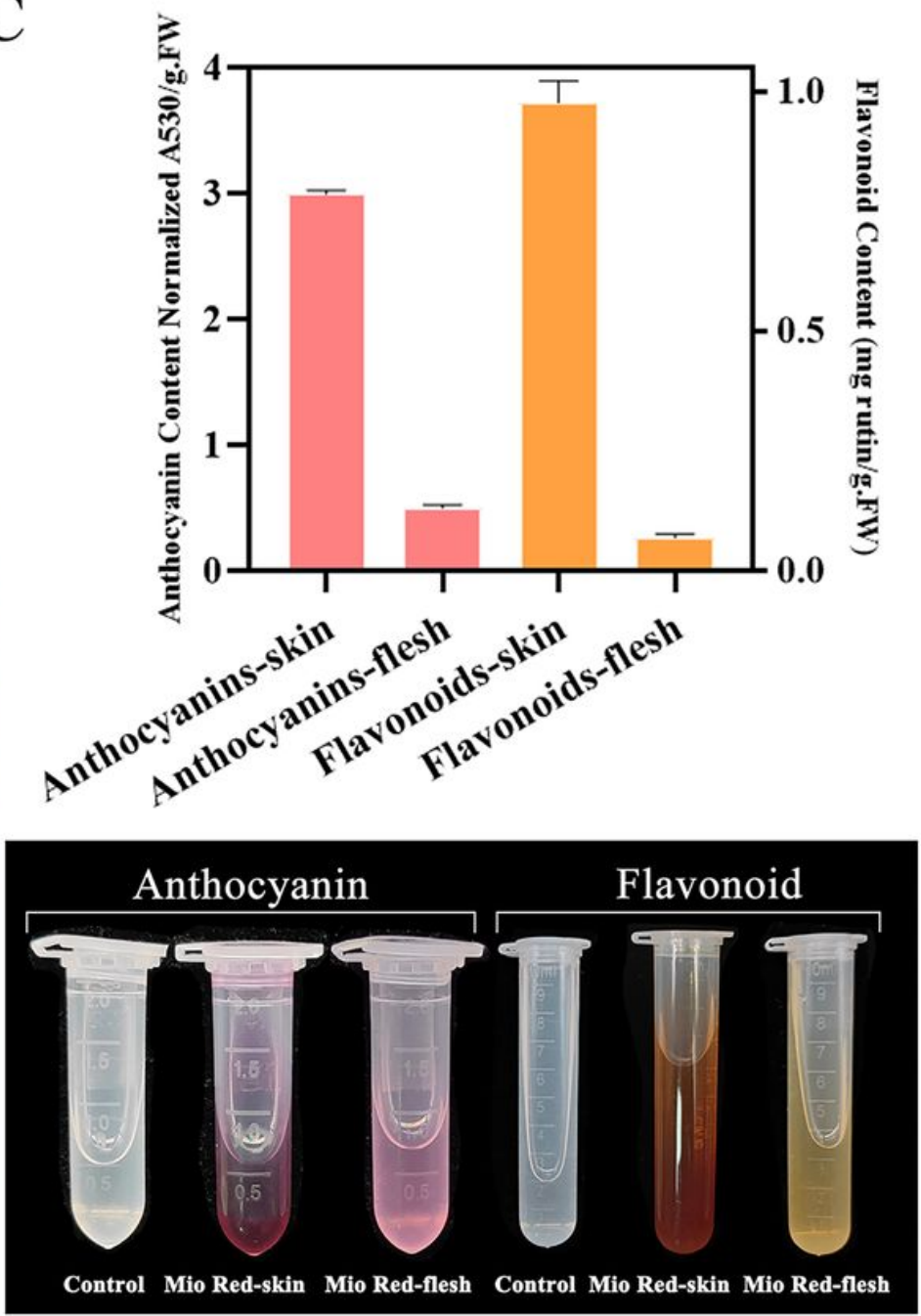

Figure 1 
Anthocyanin accumulation and total flavonoids in the skin and flesh of 'Mio Red'. (A) Coloring of the skin and flesh at berry ripening. (B) Distribution of anthocyanin in the flesh. (C) Content of anthocyanin and total flavonoids in the skin and flesh
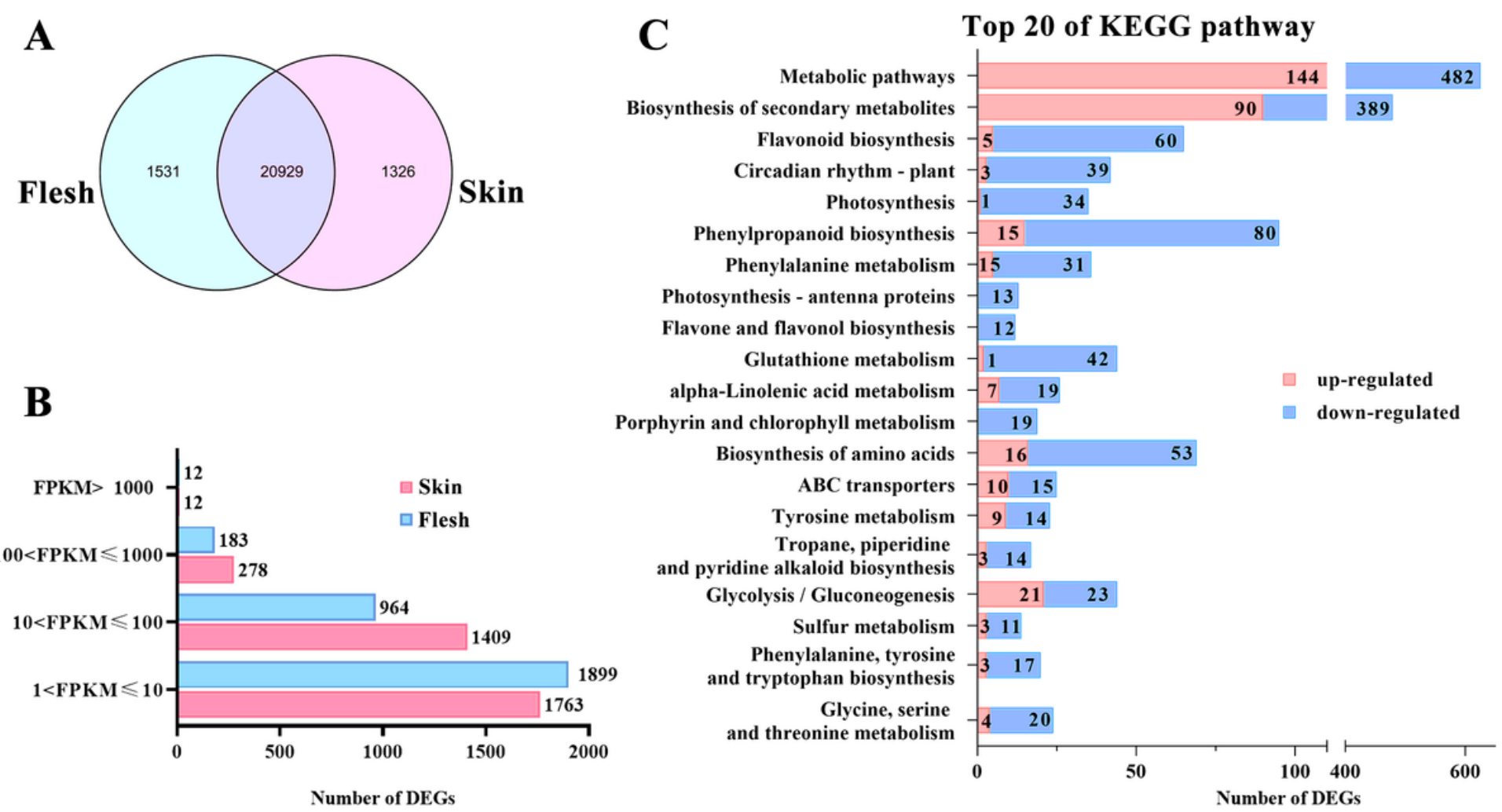

Figure 2

Transcriptome analysis of the skin and flesh of 'Mio Red' grape. (A) Venn diagram of DEGs of the skin and flesh. (B) FPKM value distribution of DEGs of the skin and flesh. (C) Enriched KEGG pathways (TOP20) 


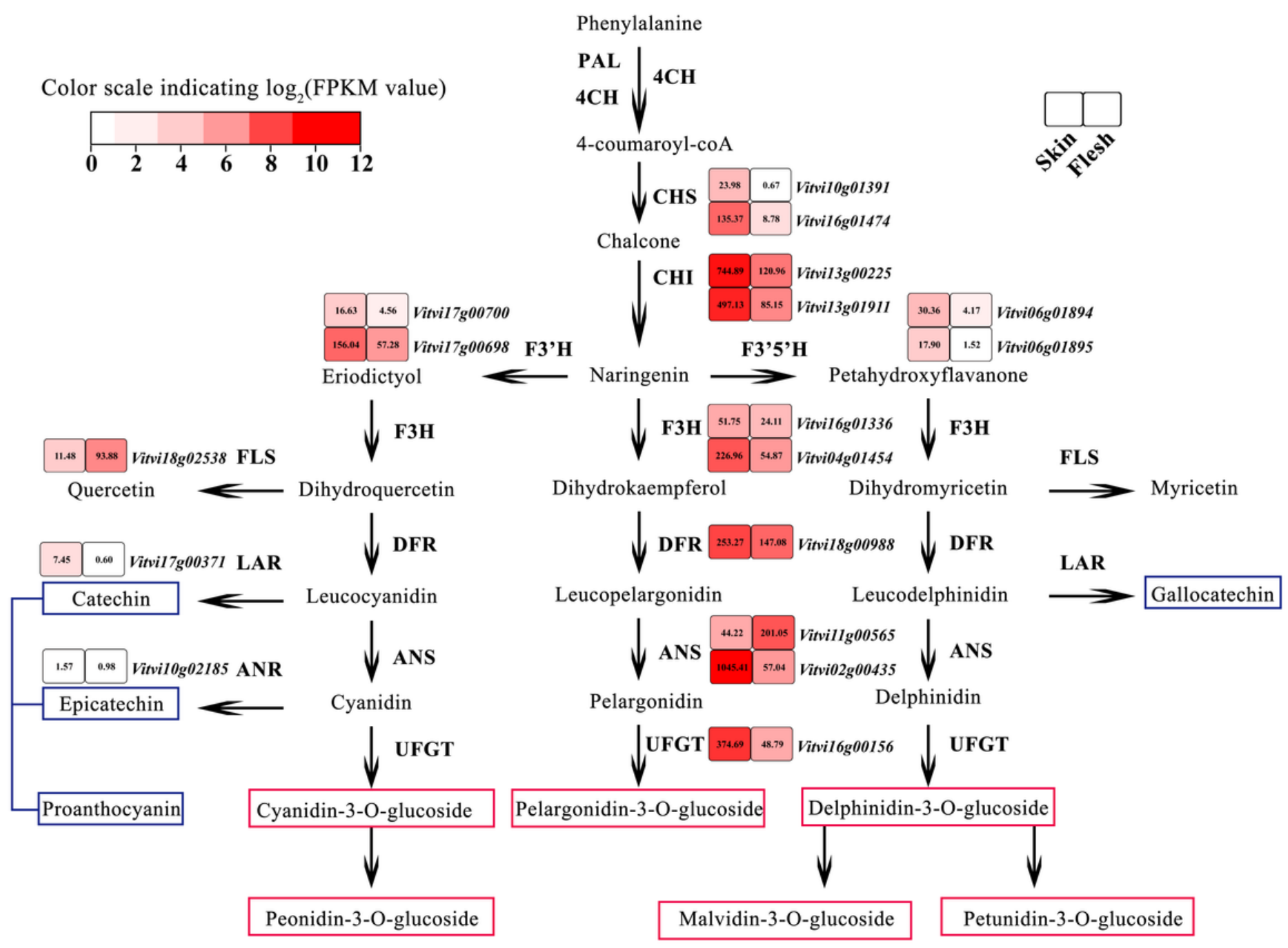

Figure 3

Expression profiles of DEGs involved in flavonoid biosynthesis pathway. The scale from white (low) to red (high) indicates FPKM value in skin and flesh of 'Mio red' 

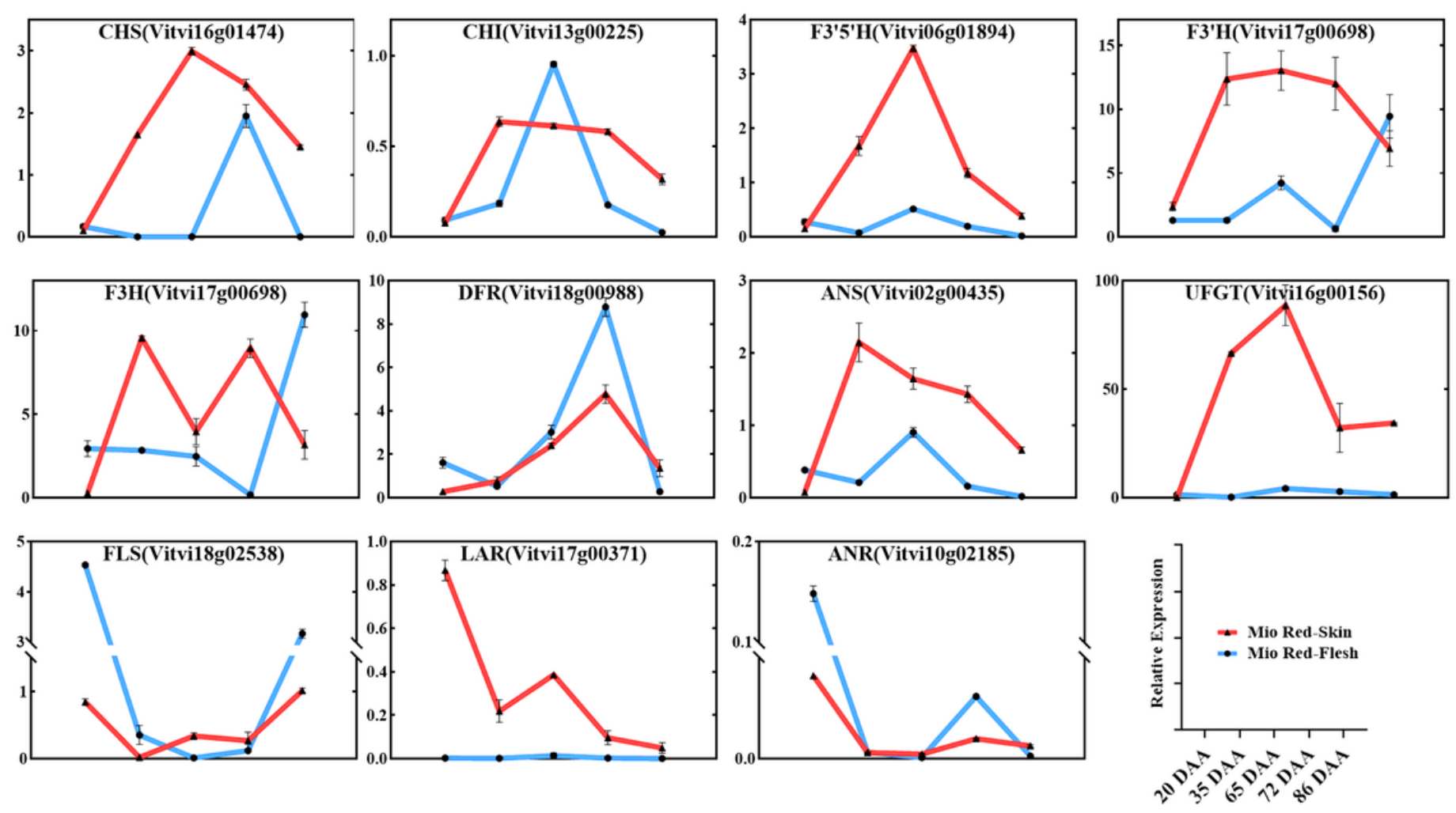

Figure 4

Expression analysis of structural genes in flavonoid biosynthesis pathway during berry development.

DAA: days after anthesis. The expression levels were normalized to mean \pm SE of 3 biological replicates 

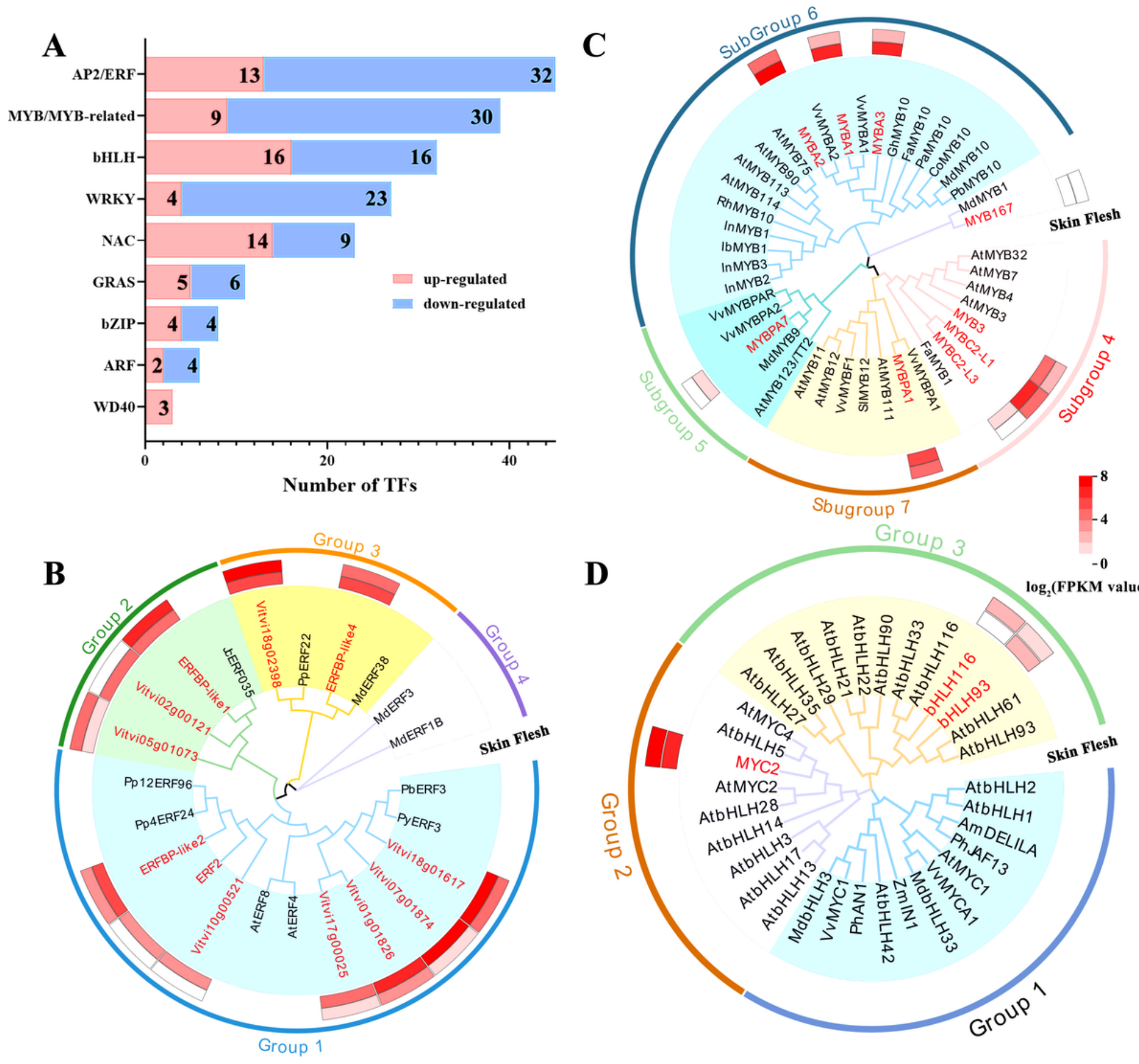

\section{Figure 5}

Clustering of differentially expressed TFs in the skin and flesh of 'Mio Red'. (A) The expression style of major TF families. (B) Phylogenetic tree analysis of ERF DEGs. (C) Phylogenetic tree analysis of R2R3-

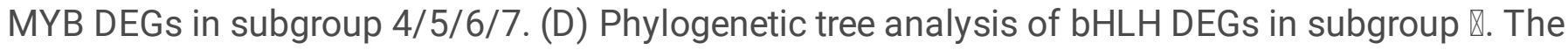
GenBank accession numbers of the TFs can be found as supplementary Table 9 
A
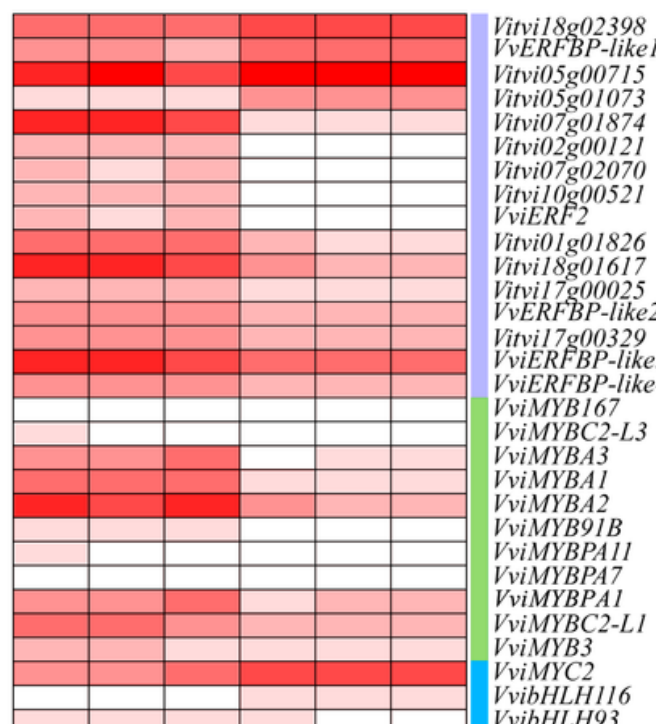

Vitvi05g00715

Vitvi05g01073

Virvi07g01874

Vitvi02g00121

itvi07g02070

Vitvilog0052

VVIERF2

Vitvi01g01826

Vitvil8g01617

Vitvil $7 \mathrm{~g} 00025$

vERFBP-like 2

Vitvil $7 g 00329$

viERFBP-like

VIMYB 167

ViMYB 167

ViMYBC2-L3

VIMYBA3

VviMYBAI

VviMYBA2

VviMYB91B

VviMYBPA11

VviMYBPA7

VIMYBPAI

VIMYBC2-LI

VviMYB3

VviMYC2
VvibHLHIl

VvibHLH 93

VVINACIOO

VviNAC71

VviNAC83

VviNAC42

VviNAC42
VviNAC68

VviNAC68 8

VvibZIP4I
VvibZIP9

Vitvi04g01136

Vitvilog01520

Vitvil3g01226

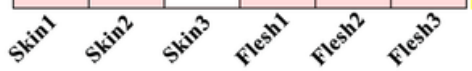

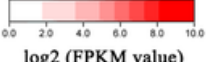

110 뭉

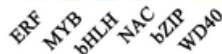

B
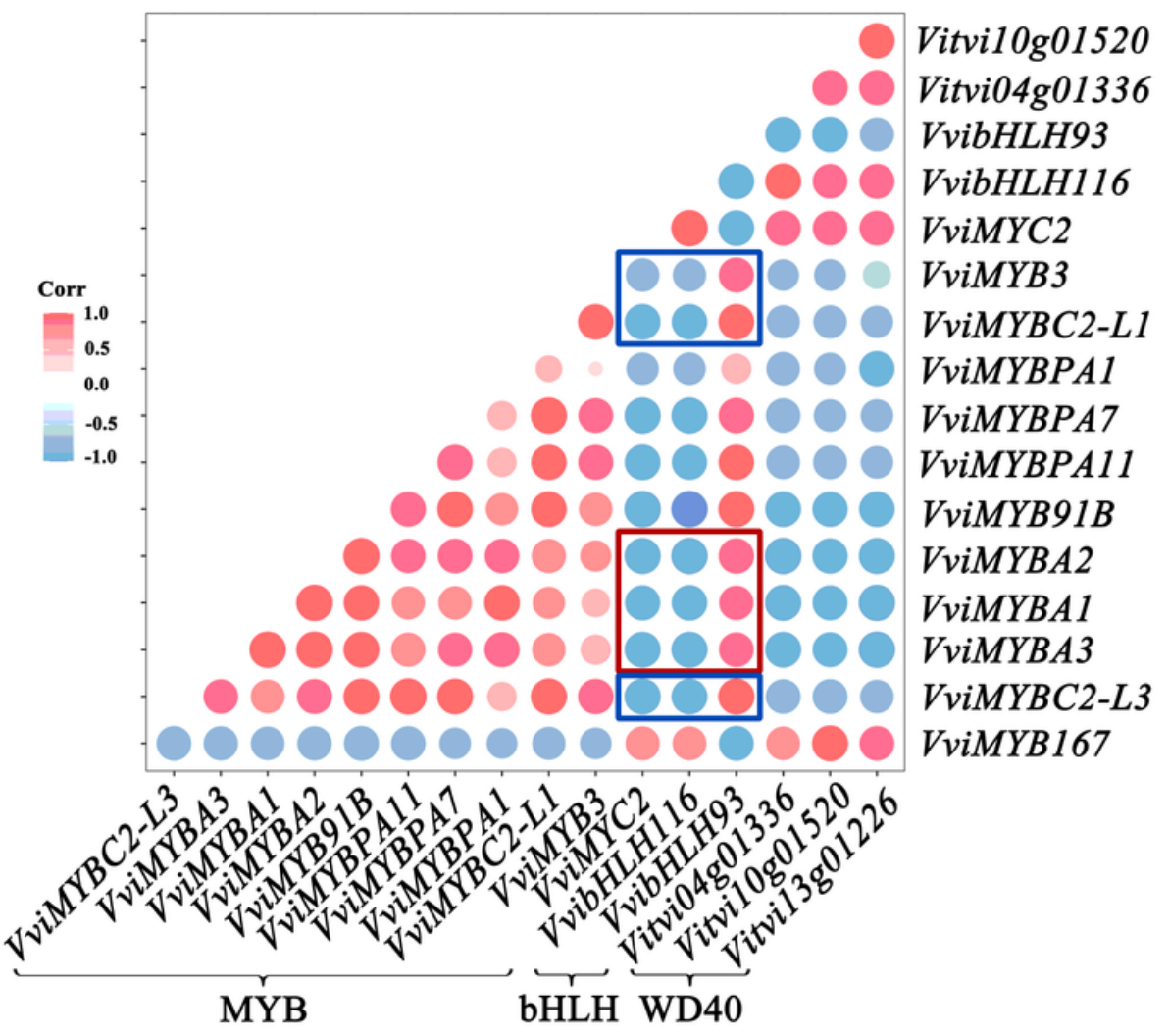

Figure 6

Expression profiles of flavonoids-related TFs and correlation analysis of MBW complex in the skin and flesh of 'Mio Red'. (A) Expression profiles of screened TFs from different family. (B) MYB-bHLH-WD40 expression correlation heatmap. Red indicates positive correlation, blue indicates negative correlation, and the size of circle indicates significant level of the correlation 

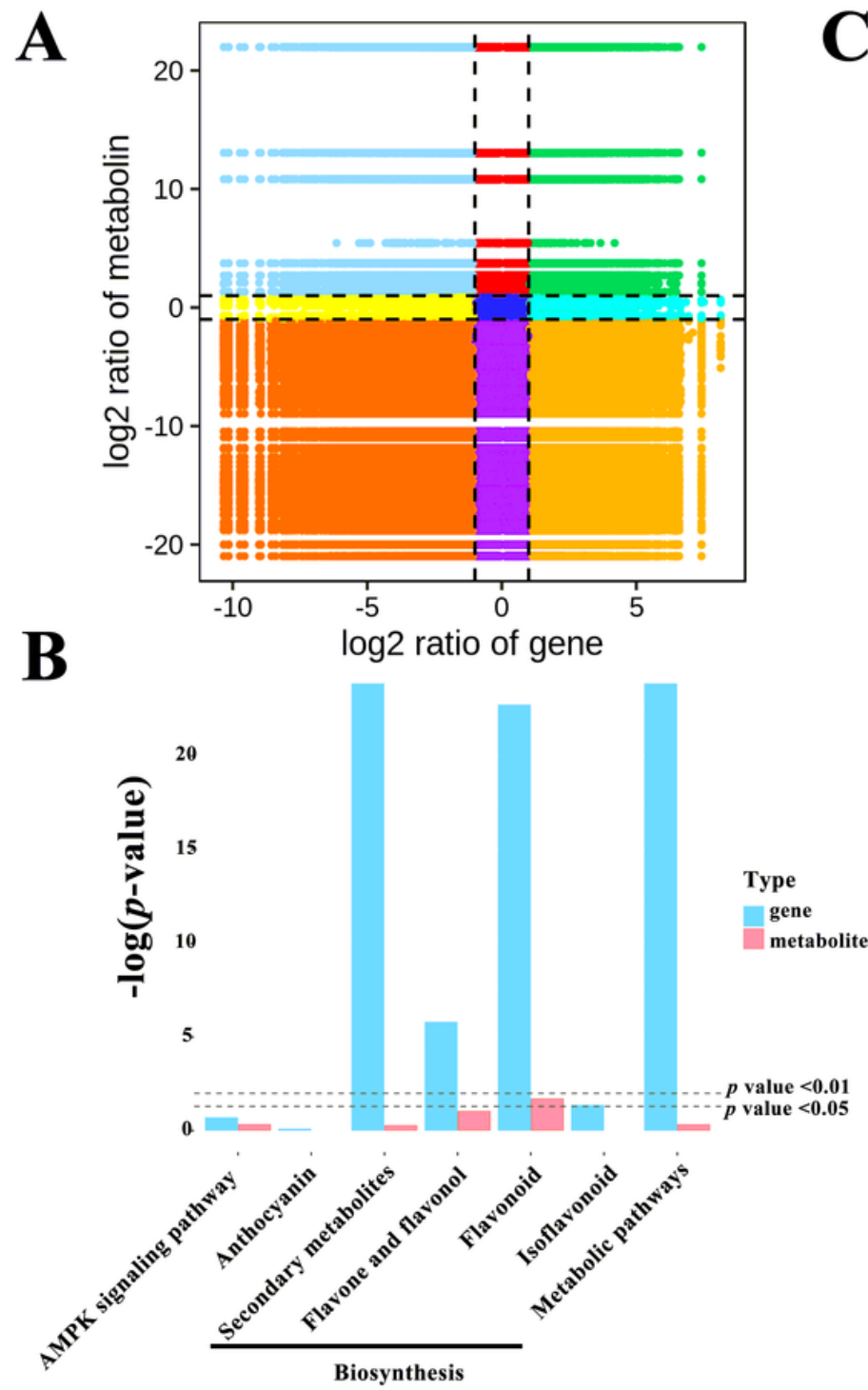

Color scale indicating $\log _{2}($ FPKM value) Circle size indicating significance level 0.02 .04 .06 .08 .010 .012 .0 $\bigcirc \bigcirc \bullet \bullet \bullet \bullet \bigcirc \bigcirc$

GST1 00000000000000000 GST2 00000000000000000 GST3 00000000000000000 GST4 $\bigcirc \circ \bigcirc \bigcirc 00000000$ GST5 0000000000000000 GST6 0000000000000000 GST7 0000000000000000

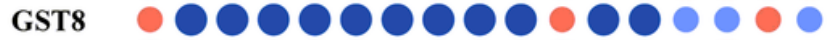
GST9 00000000000000000 GST10 0000000000000000 GST11 00000000000000000 GST12 00000000000000000 GST13 0000000000000000 GST14 0000000000000000 GST15 ○ MATE1 $\circ \circ 0 \circ \circ \circ \circ \circ \circ \circ \circ \circ$

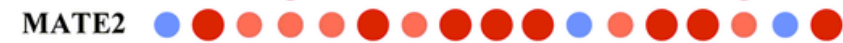

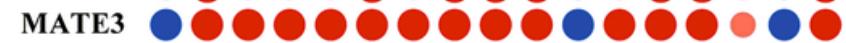
MATE4 00000000000000000 MATE5 $\bigcirc \circ \bigcirc \bigcirc \bigcirc \bigcirc \bigcirc \bigcirc \bigcirc \bigcirc \bigcirc \bigcirc 0 \bigcirc \bigcirc$ ABCB1 $\circ 00 \div$ ABCC2-1 00000000000000000 ABCC2-2 00000000000

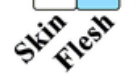

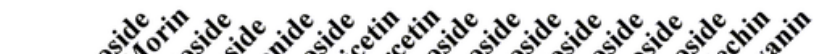

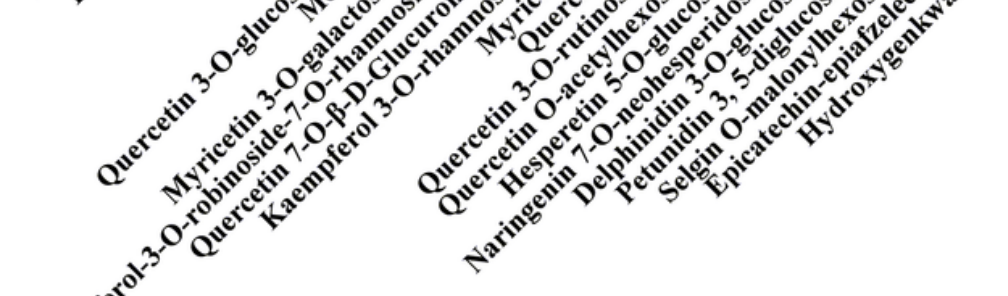

Figure 7

Integral analysis of DAMs and DEGs. (A) Nine-quadrant diagram, DEGs and DAMs with Pearson correlation coefficients greater than 0.8 were divided into 1-9 quadrants from left to right and from top to bottom with black dotted lines. (B) KEGG enrichment histogram, the X-axis represents the metabolic pathways, the Y-axis represents the expression as -log ( $p$-value). Green and red represent DEGs and DAMs, respectively. (C) Expression profile of flavonoids-associated transporters and their correlation heatmap with DAMs. Red presents positively correlated, blue presents negatively correlated, and the size of the circle indicates significance. The larger the circle, the higher the significance

\section{Supplementary Files}

This is a list of supplementary files associated with this preprint. Click to download.

- SupplementaryTables.xlsx 
- Supplementaryfigure1109.pdf 